\title{
Clinical Study of Type 2 Diabetes Mellitus in Women with Comorbidities
}

\author{
${ }^{1}$ Mamatha B Patil, ${ }^{2}$ Faris UK Padikkal, ${ }^{3}$ Gowri Ramesh
}

\begin{abstract}
Introduction: There are evidence-based guidelines for the management of diabetes mellitus (DM). These will not take into account, comorbidity the patient has, and these may come in the way of diabetes management. There are a wide range of comorbidities in Type 2 diabetes (T2DM) which again depends on the study subjects selected and the accompanying diseases.
\end{abstract}

Methodology: This cross-sectional, observational project was conducted in Rajarajeswari Medical College and Hospital (RRMCH) which included 96 women above the age of 30 years to analyze the number of comorbidities associated with diabetes mellitus. These women were selected based on the criteria and detailed history, and lab investigations were done and tabulated.

Results: Based on the investigations it is found that $67.7 \%$ of patients were hypertensive. Thirteen patients had hypothyroidism, 11 patients had dislipidemia and 14 patients had nonalcoholic fatty liver disease (NAFLD). There were 18 patients without any comorbidity in our study.

Conclusion: It was observed that (a) Diabetes mellitus is most commonly associated with hypertension as the comorbidity. (b) As the age advances risk to comorbidities also increases.

Keywords: Comorbidities, Hypertension, Type 2 diabetes mellitus

How to cite this article: Patil MB, Padikkal FUK, Ramesh G. Clinical Study of Type 2 Diabetes Mellitus in Women with Comorbidities. J Med Sci 2018;4(3):87-89.

Source of support: Nil

Conflict of interest: None

\section{INTRODUCTION}

Diabetes mellitus (DM) belongs to a group of metabolic diseases associated with hyperglycemia due to either defect in insulin secretion and insulin action or both. Diabetes mellitus can be classified as Type 1 (insulin-dependent) and 2 (non-insulin dependent) diabetes mellitus.

\footnotetext{
${ }^{1}$ Professor, ${ }^{2}$ Junior Resident, ${ }^{3}$ House surgeon

${ }^{1-3}$ Department of General Medicine, RajaRajeswari Medical College Hospital, Bengaluru, Karnataka, India

Corresponding Author: Mamatha B Patil, Professor, Department of General Medicine, RajaRajeswari Medical College Hospital, Bengaluru, Karnataka, India, e-mail: Dr.mamatharamesh@ yahoo.in
}

Diabetes mellitus (DM) affects 382 million people worldwide, and its prevalence is ever increasing, the prediction being a rise to 592 million by the year $2035 .{ }^{1}$ According to the World Health Organization (WHO), it is predicted that by $2030 \mathrm{DM}$ may affect up to 79.4 million. It is also predicted that the urban population in developing countries will increase from 1.9 billion in 2000 to 3.9 billion in 2030.

When we consider diabetes as most prevalent condition, any condition that may co-exist is considered comorbidity. It is said that in primary health care, more than $40 \%$ of patients with DM will have comorbidities. It ranges from 70 to $95 \%$ in the literature pertaining to DM cohorts studies. DM with comorbidity has a negative effect on quality of life, and it substantially increases their health care utilization and cost. ${ }^{2}$

There are a few studies on comorbidity in DM. ${ }^{3}$ Recommendations for clinical approaches insufficiently provided in evidence-based DM guidelines. ${ }^{4}$

Hence we conducted a cross-sectional study to assess the proportion of women with comorbidities associated with T2DM.

We also estimated the number of comorbidities in women $>30$ years with known and recently diagnosed T2DM.

We also found out the common comorbidity affecting women above the age of 30 years.

\section{METHODOLOGY}

\section{Study Design}

\section{An Observational Clinical Study}

Patients were recruited as per the inclusion criteria. Only subjects who meet requirements in this section, having signed an informed consent with the investigator will be recruited in this cross-sectional study.

\section{Inclusion Criteria}

- All female patients aged above 30 years who are known cases or newly diagnosed cases of T2DM.

- All female patients with T2DM attending medicine OPD and admitted in all-female medical wards at $\mathrm{RRMCH}$

- Patients with or without known comorbid illnesses are included. 


\section{Exclusion Criteria}

- Pregnant women with gestational DM.

- Patients with Type 1 DM

- Patients not willing for examination and investigations and not signing informed consent with the investigator.

\section{Methodology}

- Patients were subjected to detailed history, clinical examination and specific lab investigations. The case summary was documented in the proforma and tabulated.

- Demographic data and socioeconomic status, detailed history related to diabetes, symptoms, drug history, family history, personal history, obstetric and menstrual history and history suggestive of any comorbid illnesses.

- Detailed clinical examination including systemic examination.

- Lab investigations were done in all patients which included complete hemogram, FBS, PPBS, HbA1C, lipid profile, BUN, serum creatinine and urine routine examination. TFT in suspected or known cases of thyroid disorders. USG abdomen, CT, MRI, ECG, $\mathrm{ECHO}$ as and when indicated. The patients were followed till the completion of all investigations.

\section{Statistical Methods}

Continuous measurements are presented on mean \pm SD (Min-max), and results on categorical measurements are presented in Number (\%). The significance is assessed at $5 \%$. Analysis of variance (ANOVA) has been used to find the significance of study parameters between three or more groups of patients,

Chi-square/Fisher's exact test has been used to find the significance of study parameters on a categorical scale between two or more groups, the non-parametric setting for qualitative data analysis. Fisher's exact test used when cell samples are very small. The statistical software Statistical Package for the Social Sciences (SPSS) 18.0, and the $R$ environment version 3.2.2 were used for the analysis of the data and Microsoft Word and Excel have been used to generate graphs, tables, etc.

\section{RESULTS}

The average age of the patients was 56.64 years. Among 96 patients, 62 patients were above 50 years of age, and 34 patients were below 50 years of age (Table 1 ).

Among the total number of 96 patients studied 78 patients had comorbidities, and 18 patients had no comorbidities (Table 2).
In the study 65 patients were hypertensive, 13\% patients had hypothyroidism, $11 \%$ patients had dyslipidemia, and $14 \%$ patients had NAFLD. Hypertension was the commonest comorbidity in our study. $67.7 \%$ patients were hypertensive (Table 3).

Forty-seven patients have comorbidities between the age of 30 to 60 and 31 patients have comorbidities between the age of 60 and 80 (Table 4).

In our study there were 59 patients with only one comorbidity, 15 patients with two comorbidities, 3 patients with 2 comorbidities and 2 patients with all four comorbidities, There were 18 patients without any comorbidity in our study (Table 5).

In our study, the average age of patients who had all four comorbidities were 63 years, and 57.9 years who had only one comorbidity.

This indicates that as age advances comorbidities also increases (Table 6).

\section{DISCUSSION}

T2 DM is one of the metabolic disorders, characterized by hyperglycemia which may be due to either insulin resistance or relative insulin deficiency. ${ }^{5}$ It is a multiorgan chronic disease and is associated with a ten-year-shorter life expectancy due to its complications. ${ }^{6}$ As per the rough estimate, 415 million adults have diabetes and it may increase to 642 million by the year 2014. The incidence of Type 2 diabetes is increasing in several countries.

Table 1: Distribution of age groups

\begin{tabular}{lll}
\hline Age (years) & No. of patients & Percentage \\
\hline $30-40$ & 11 & 11.5 \\
$41-50$ & 23 & 24.0 \\
$51-60$ & 28 & 29.2 \\
$61-70$ & 27 & 28.1 \\
$71-80$ & 7 & 7.3 \\
Total & 96 & 100.0 \\
\hline
\end{tabular}

Mean \pm SD: $56.64 \pm 11.49$

Table 2: Incidence of comorbidities and their distribution

\begin{tabular}{lll}
\hline Risk of comorbidity & No. of patients & Percentage \\
\hline & No. of patients & Percentage \\
No & 18 & 18.8 \\
Yes & 78 & 81.3 \\
Total & 96 & 100.0 \\
\hline
\end{tabular}

Table 3: Incidence of comorbidity conditions

\begin{tabular}{lll}
\hline & No. of patients $(n=96)$ & Percentage \\
\hline HTN & 65 & 67.7 \\
Hypothyroid & 13 & 13.5 \\
Dyslipidemia & 11 & 11.5 \\
NAFLD & 14 & 14.6 \\
\hline
\end{tabular}


Clinical Study of Type 2 Diabetes Mellitus in Women with Comorbidities

Table 4: Incidence and risk of comorbidities in different age groups and their distribution

\begin{tabular}{llll}
\hline \multirow{2}{*}{ Age (years) } & \multicolumn{3}{c}{ Risk of comorbidity } \\
\cline { 2 - 4 } $30-40$ & No & Yes & Total \\
$41-50$ & $7(38.1 \%)$ & $9(11.5 \%)$ & $11(11.5 \%)$ \\
$51-60$ & $6(33.3 \%)$ & $16(20.5 \%)$ & $23(24 \%)$ \\
$61-70$ & $3(16.7 \%)$ & $24(30.8 \%)$ & $27(28.1 \%)$ \\
$71-80$ & $0(0 \%)$ & $7(9 \%)$ & $7(7.3 \%)$ \\
Total & $18(100 \%)$ & $8(100 \%)$ & $96(100 \%)$ \\
Mean \pm SD & $53.33 \pm 9.37$ & $57.40 \pm 11.85$ & $56.64 \pm 11.49$ \\
\hline
\end{tabular}

$p=0.177$, Not significant, Student t-test
Table 5: Number of comorbiditiess

\begin{tabular}{lll}
\hline $\begin{array}{l}\text { Number of } \\
\text { comorbidities }\end{array}$ & No. of patients & Percentage \\
\hline 0 & 18 & 18.8 \\
1 & 59 & 61.5 \\
2 & 15 & 15.6 \\
3 & 2 & 2.1 \\
4 & 2 & 2.1 \\
Total & 96 & 100.0 \\
\hline
\end{tabular}

Mean \pm SD: $56.64 \pm 11.49$

Table 6: Comparison of age according to the risk of comorbidity

\begin{tabular}{|c|c|c|c|c|c|c|}
\hline \multirow[b]{2}{*}{ Age in years } & \multicolumn{5}{|c|}{ Risk of comorbidity } & \multirow[t]{2}{*}{ Total } \\
\hline & Nil & 1 & 2 & 3 & 4 & \\
\hline Age in years & $53.33 \pm 9.37$ & $57.97 \pm 12.63$ & $53.73 \pm 9.06$ & $62.50 \pm 3.54$ & $63.00 \pm 7.07$ & $56.64 \pm 11.49$ \\
\hline
\end{tabular}

$p=0.393$, Not significant, ANOVA test

Low- and middle-income countries have a higher incidence of DM. The higher incidence with DM is in the age groups of 40 and 59 years. Five million deaths in 2015 were caused by DM, and it is said that every six seconds a person dies from diabetes. In the US 673 billion dollars are spent to treat $\mathrm{DM}$, and it is $12 \%$ of the of the budget on health expenditure. It is estimated that over 542,000 children suffered from Type 1 diabetes in 2015. One in 7 live births was affected by DM. ${ }^{7}$ It is predicted that by the year 2025, India, China, and the US ${ }^{8}$ will have the largest number of population with diabetes.

Our objective was to assess the proportion of women with comorbidities associated with T2DM, to estimate the number of comorbidities in women $>30$ years with known and recently diagnosed T2DM and to find out the commonest comorbidity affecting women above the age of 30 years.

There is a limited number of studies on comorbidity in DM. In the literature, previous studies have focused mainly on comorbid conditions that share pathogenesis, risk factors, and management plans with DM (e.g., hypertension) In some diseases without shared pathogenesis, the incidence of risk factors or management, have not been studied. Both groups of diabetes patients show similar increases in cost and healthcare utilization. Recommendations for clinical approaches to both these groups are insufficiently provided in evidence-based DM guidelines.

\section{CONCLUSION}

Our study included 96 women above the age of 30 years. This study was conducted to analyze the common comorbidities associated with diabetes mellitus in women.
Among the total number of 96 patients studied 78 patients had comorbidities, and 18 patients had no comorbidities (Table 2).

Hypertension was the most frequent comorbidity in our study. About $67.7 \%$ of patients were hypertensive (Table 3). Thirteen patients had hypothyroidism, 11 patients had dyslipidemia, 14 patients had NAFLD. In our study, there were 59 patients with only one comorbidity, 15 patients with two comorbidities, 2 patients had three comorbidities and 2 patients with all four comorbidities (Table 5). There were 18 patients without any comorbidity in our study. There were 18 patients without any comorbidity in our study. It was also observed that as the age advances (Tables 4 and 6), the risk to comorbidities also increases.

\section{REFERENCES}

1. Seuring T, Archangelidi O, Suhrcke M. The economic costs of type 2 diabetes: a Global Systematic Review. PharmacoEconomics. 2015;33:811.

2. Politis $\mathrm{M}$, Lindvall O. Clinical application of stem cell therapy in Parkinson's disease. BMC Medicine. 2012;10:1.

3. OH Oduaran (2015).

4. Rosner B. Fundamentals of Biostatistics, 5th edition. New Delhi: Duxbury (Cengage); 2000. pp. 80-240.

5. American Diabetes Association. Diagnosis and Classification of Diabetes Mellitus. Diabetes Care. 2014;37(Suppl 1): S81-S90.

6. Meetoo D, McGovern P, Safadi R. An epidemiological overview of diabetes across the world. Br J Nurs. 2007;16(16):1002-7.

7. International Diabetes Federation. IDF Diabetes Atlas, 7th edition. Brussels: IDF; 2015

8. King H, Aubert RE, Herman WH. Global Burden of Diabetes, 1995-2025: Prevalence, numerical estimates, and projections. Diabetes Care. 1998;21(9):1414-1431. 